\title{
DRIVER FATIGUE AND DROWSINESS DETECTION SYSTEM
}

\author{
MVS Roja Ramani \\ Asst. Professor \\ NSRIT, Visakhapatnam, India \\ Allu Tejaswee, P.S.T.B. Gupta, Kalepu Harikrishna, Ganisetti Usha Sri \\ Student, ECE \\ NSRIT, Visakhapatnam, India
}

\begin{abstract}
In present days, automobile industries share a numerous accidents due to the distractions such as fatigue and lack of sleep. These accidents can be avoided by keeping a keen view on the tired characters of driver a producing an alert when the driver is found to be drowsiness state.
\end{abstract}

\section{Keywords- OpenCV, Python, Numpy, Scipy, Pygame}

\section{INTRODUCTION}

Due to the increase in automobile development that has been taking place in recent years, the accidents that were taking place has been increased and the problems created by them are complex as well. The investigation reports prove that these were occurred due to the drunk, drowsiness behaviour of the driver. So, it is necessary to develop an "Driver Fatigue and Drowsiness Detection System" which improves the driver safety.

Driver Fatigue is the important factor that has to be considered in road accidents. The recent statistics say that annually there were more than 1,500 accidents which were occurred only due to the fatigue behaviour of the driver. The lead to the fatigue crashes. The development of technology that can detect and prevent the drowsiness is the major challenge in accidents avoiding systems. The major aim of this project is to create a fatigue alertness system. The main focus is to find the tired characters of the driver and generate an alert when the driver is found to be drowsy state. It has to monitor the open and close movements of eye of the driver in real-time. By keenly observing and generating an alert we can prevent the road accidents which are caused due to drowsiness.
Detection of this involves reading of number of images of the face and observing the eyelid movements and eye blink rate. This analysis is popular with applications such as face recognition. The project is mainly focused on localising the eyes. This involves looking at the face in the image entirely and finding the location of the eyes in that particular image. If once the eye location is determined then the system is designed in particular way that it can find the drowsiness of the driver.

Driver Fatigue and Drowsiness detection is in existence from past 5years. As the first model, the system is designed with the Arduino and IR sensors. The sensors are attached to the

Spectacles of the person, when the person closes his eye the receiver in the IR cannot get the rays from the transmitter. As a result, the alarm which is programmed to activate when the eyes are closed used to produce a sound for alerting the person. The results in this proposal were inaccurate and lead to the disadvantages of this system.

Later, the Drowsiness Detection System was implemented using MATLAB. In this project, the image can be processed using MATLAB. But the problem is, when it is working on real time frames from the image. But there was a problem in this design, the processing time by this design is very high. Due to that the model was a failure for real-time.

To overcome this problem, the solution is obtained by designing this project by OPENCV and DLib libraries which can be used in real-time projects.

There were several types of systems to design the drowsiness detection system. But possible methods are: Sensing the psychological state of the driver, 


\section{International Journal of Engineering Applied Sciences and Technology, 2020 \\ Vol. 5, Issue 3, ISSN No. 2455-2143, Pages 231-236 \\ Published Online July 2020 in IJEAST (http://www.ijeast.com)}

sensing the vehicle condition, and monitoring the driver's response.

\section{SOFTWARE REQUIREMENTS}

The tools used in the project are Python, OpenCv for face detection algorithms, Numpy for numerical calculations and PyGame for generating and alert when the driver is drowsy.

\section{PYTHON:}

Python is a general purpose language which is object-oriented programming language. Python was created by Guido Van Rossum during the year 19851990. The source code if python is available under General Public Liscence (GPL).

Python is a simple language when compared to other languages. Python uses basic English keywords. Whereas the other programming languages uses punctions which are at difficulty level. This made python easy and interactive $t$ the designers.

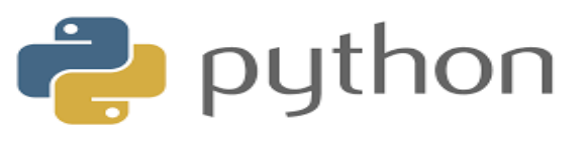

Fig 1. Python logo

\section{OpenCV:}

$\mathbf{C V}$ in OpenCv stands for "Computer Vision". It is a cross-platform library which is designed to develop several computer vision applications. OpenCV mainly focus on processing the image, capturing the video analysing and including some features like face and object detection.

Computer Vision mainly explains about how to understand a 3D from its 2D images which helps in object and face detection. It majorly deals with functioning as the human vision using the combination of computer hardware and software.

The main purpose of the computer vision is to read the content of the image and understand it. It reads the image and extract some description from it which might be an object, or text description or so on. For example, car can be built using the computer vision which helps in identifying the different objects around the car such as traffic lights, road, pedestrians and so on and work accordingly.

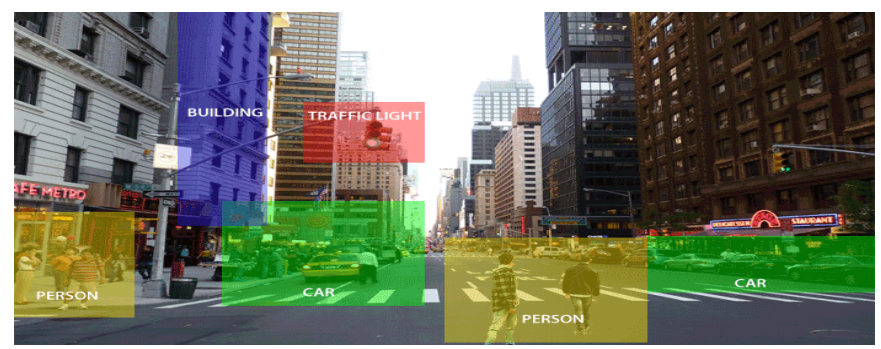

OpenCV

Fig 2. Object Detection Using the

\section{FACE DETECTION WITH OPENCV:}

OpenCV will come with a $\mathrm{n}$ detector as well as a trainer. If you were interested to train your own classifier then you can use opencv to create one.

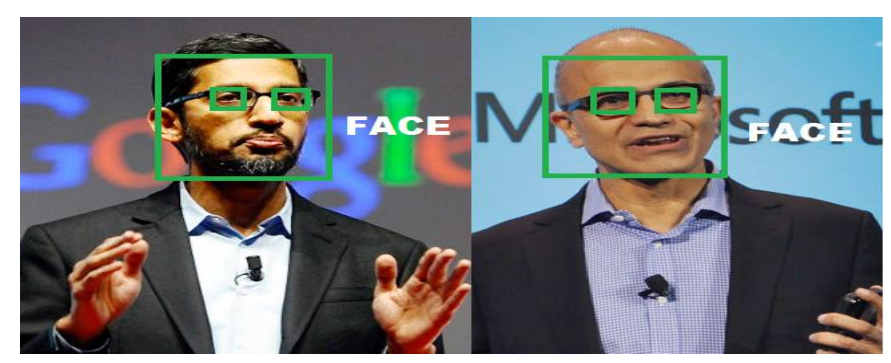

Fig 3. Eye detection using OpenCV

\section{NUMPY:}

Numpy stands for "Numerical Python". It is one of the package in python module.

Operations that can be performed by NUMPY:

Numpy can perform following operations:

- Mathematical and logical operations on arrays.

- Fourier transform for shape manipulation.

- Operations related to linear-algebra.

Numpy is the replacement of the Matlab that is used for the same mathematical operations.

\section{SciPy:}

SciPy has some basic functions to work on images. It has several functions $t$ read images to numpy arrays from disk, or to write the images on to the disk which helps in face and object detection.

\section{PyGame:}


PyGame is a python module that is designed to create video games. Pygame has excellent SDL library.

\section{BLOCK DIAGRAM}

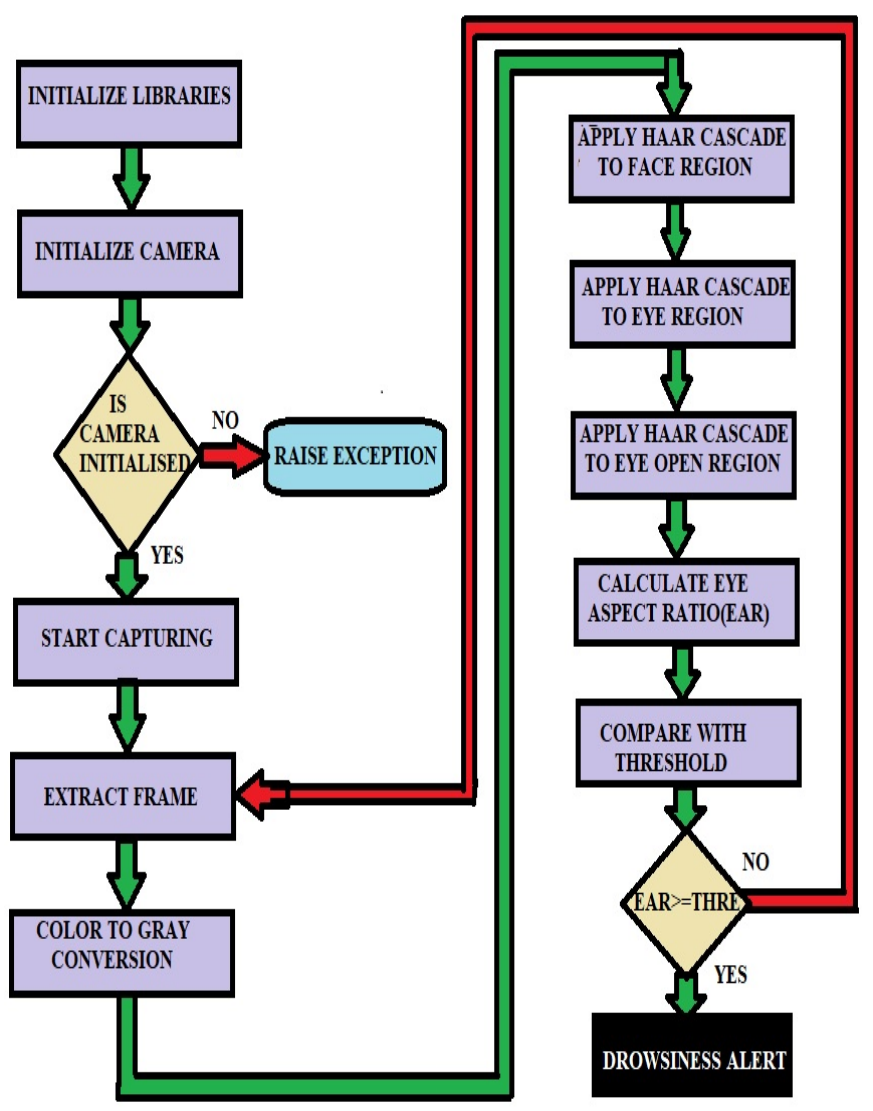

Fig 4. Block Diagram

From the above block diagram, the project can be implemented by first initialising the libraries which will help in opening the camera. Later the camera detects the face by using the opencv commands. If the camera is not initialised it raises an exception and if the camera is initialised it starts capturing. The video which has been capturing by the camera is divided into frames and the frames are converted to gray which helps to perform haar- transform on that particular images.

By applying the haar the eye aspect ratio calculated. If the EAR is greater or equals to the threshold the drowsiness alert is generated. If the EAR is lesser than the threshold then the process starts from the step-1.

\section{HARDWARE}

The hardware used in this project is webcam which is used to detect the eyelid movement of the driver and generate an alert when the driver is found to be in fatigue state.

A webcam is a video camera that is used to stream the images or video in real-time. These are small cameras that are attached to the monitor or to the hardware.

$$
\text { V. RESULTS }
$$

\section{GUI OUTPUT}

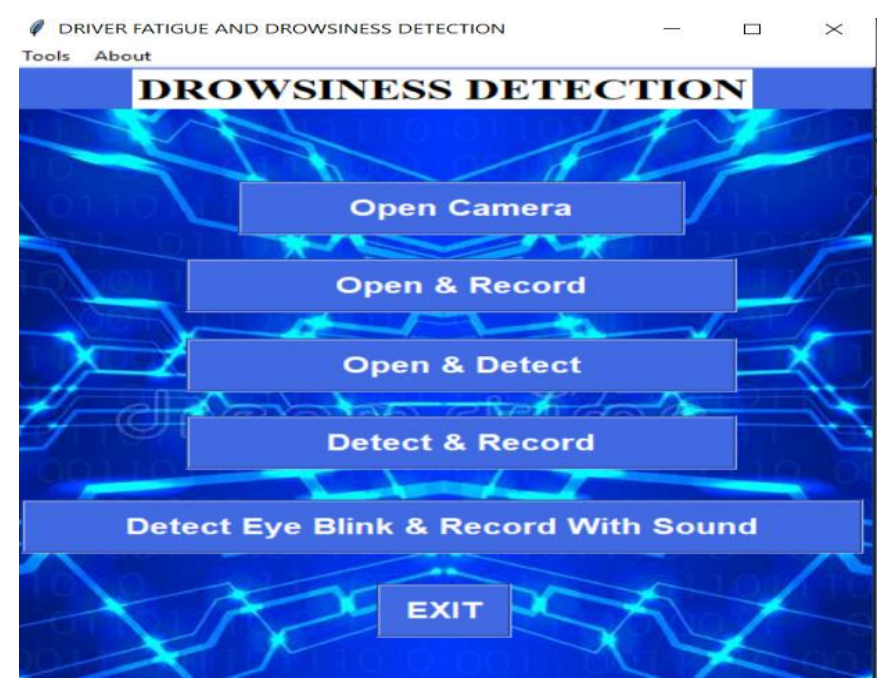

Fig 5.GUI Output

DRIVER Fatigue AND dROWSINESS DETECTION

Tools About

\section{Openc c Vocs DWSINESS DETECTION}

Figure 5 (a)Tools menu in GUI

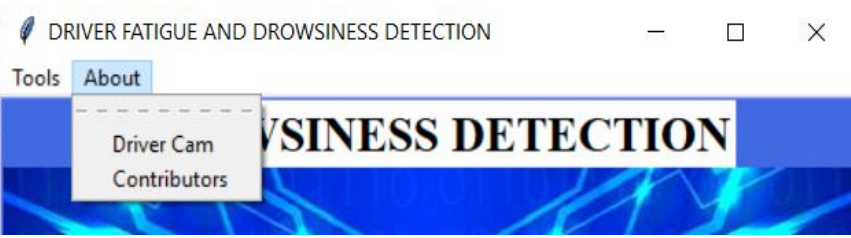

Fig. 5(b) About menu in GUI

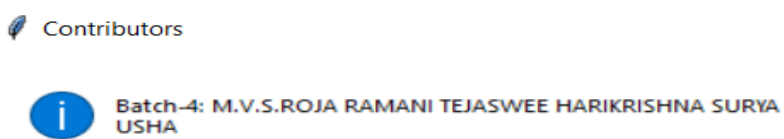

Fig. 5(c)Contributors sub menu 
International Journal of Engineering Applied Sciences and Technology, 2020

Vol. 5, Issue 3, ISSN No. 2455-2143, Pages 231-236

Published Online July 2020 in IJEAST (http://www.ijeast.com)

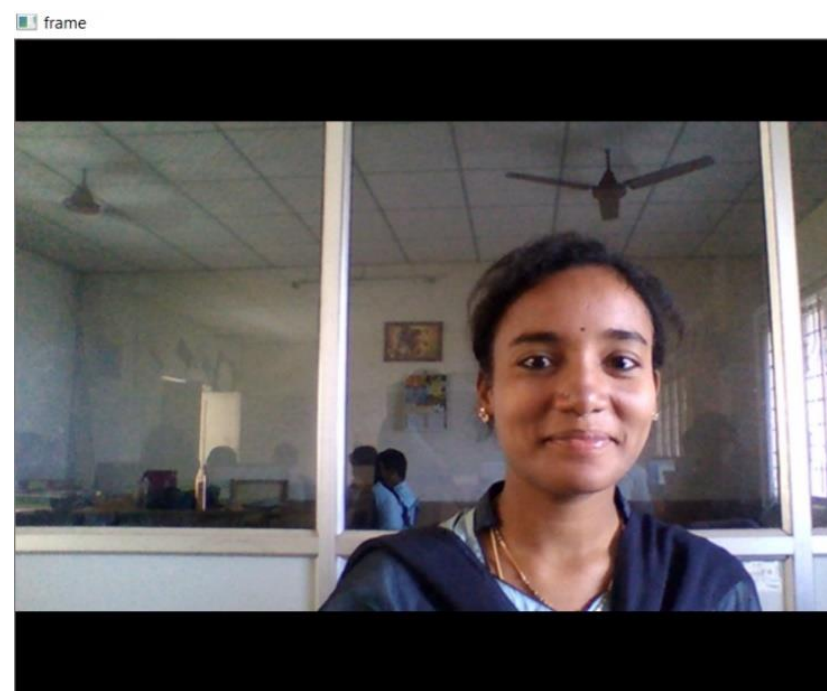

Fig 6. Open Camera

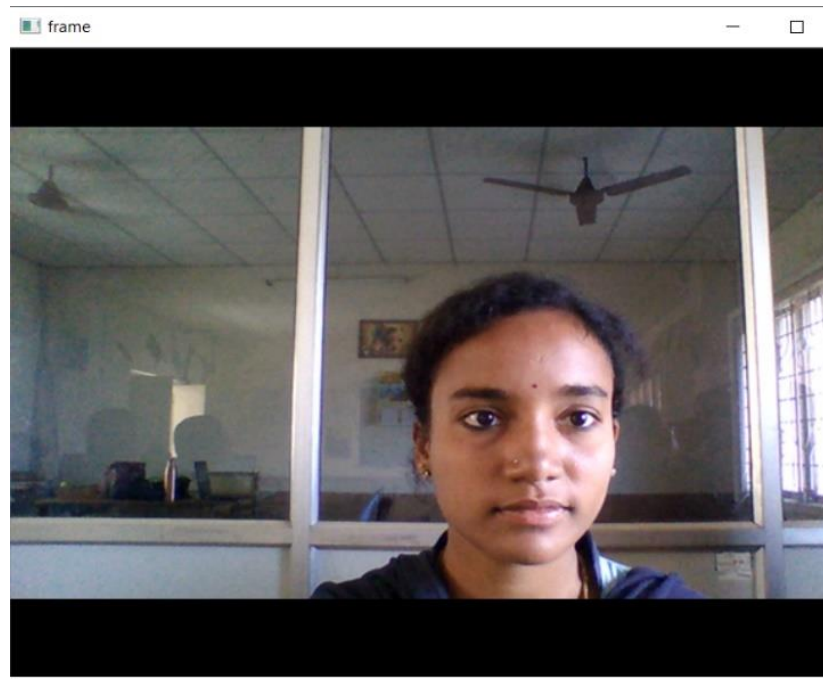

Fig 7. Open Camera \& Record
$1 \mid 01$; Drowsiness-monitoring-Using-OpenCV-Python-master

File Home share View

$\leftarrow \rightarrow \vee \uparrow \mathbb{I}$ > ThisPC > Deskiop > PROJECT > DRVVER ALERTNESSSYSTEM > Drowsiness-monitoring-Using-OpenCV-Python-master

\begin{tabular}{|c|c|c|c|c|}
\hline Ouick access & Name & Date modfied & Type & Size \\
\hline & (6) beep-07 & $16-06-201816,008$ & Wav Fle & $10 \mathrm{~KB}$ \\
\hline & I CustomBinkCascade & $16-06-201816,008$ & XML Document & $313 K B$ \\
\hline$\checkmark$ Downloods & A Driver Cam & $11 \cdot 03 \cdot 202010.15$ & Python Fle & $7 \mathrm{~KB}$ \\
\hline Documents & 9 harcascade_eye & $16-06-201816078$ & XML Document & 334 KB \\
\hline EP Pictures & I haarcascade eye tree_eyeglasses & $16-06-201816008$ & XML Document & $588 \mathrm{~KB}$ \\
\hline I Drowsiness-monitorit & $\$$ lopcascade frontallace & $16-06-201816008$ & XML Document & $51 \mathrm{~KB}$ \\
\hline I PROJECT & î new & $04-02-20201233$ & PNG Fle & $1,176 \mathrm{~KB}$ \\
\hline I PROJECT DOCUMENT & Dis PROEECT PPT & $06-03-20201227$ & Microsoft Powerpo.. & $2,759 \mathrm{~KB}$ \\
\hline I teju & READMEmd & $16-06-2018160.00$ & MDFle & $1 \mathrm{~KB}$ \\
\hline OneDrive & E) Samplel & $11-03-2020101919$ & Al File & 9,478 KB \\
\hline
\end{tabular}

9 This PC

30 Objects

Desktop

- Documents

$\checkmark$ Downloads

J Music

Fig 8. Open Camera \& Record sample output

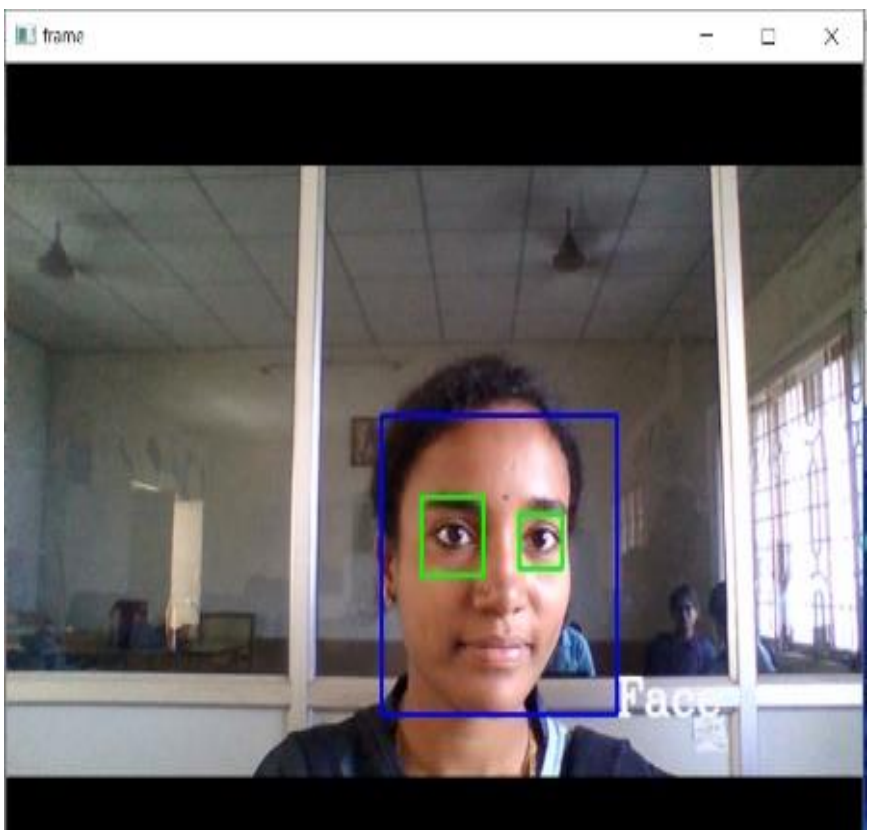

Fig 9. Open Camera \& Detect 


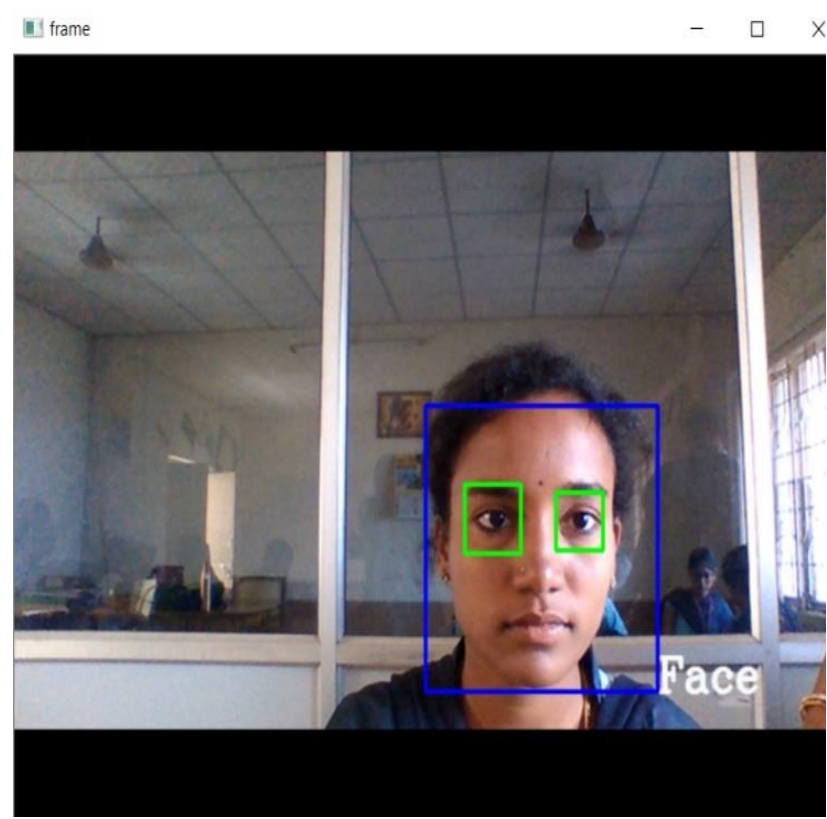

Fig 10. Detect \& Record

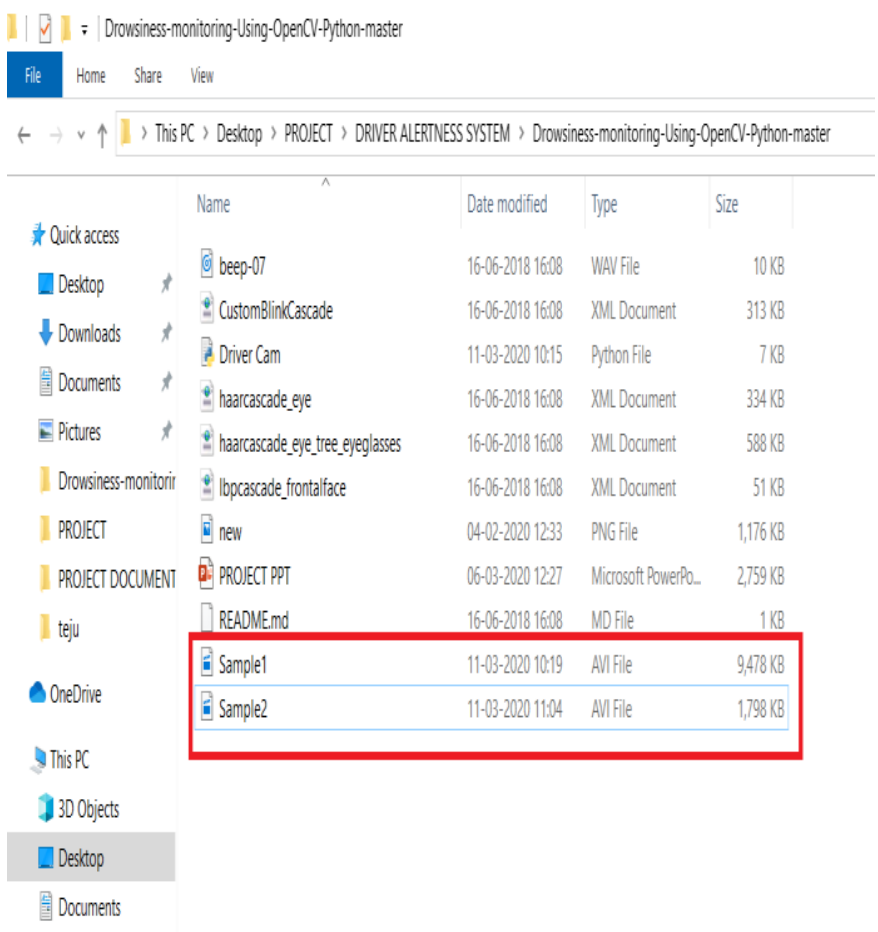

Fig 11. Detect \& Record sample output

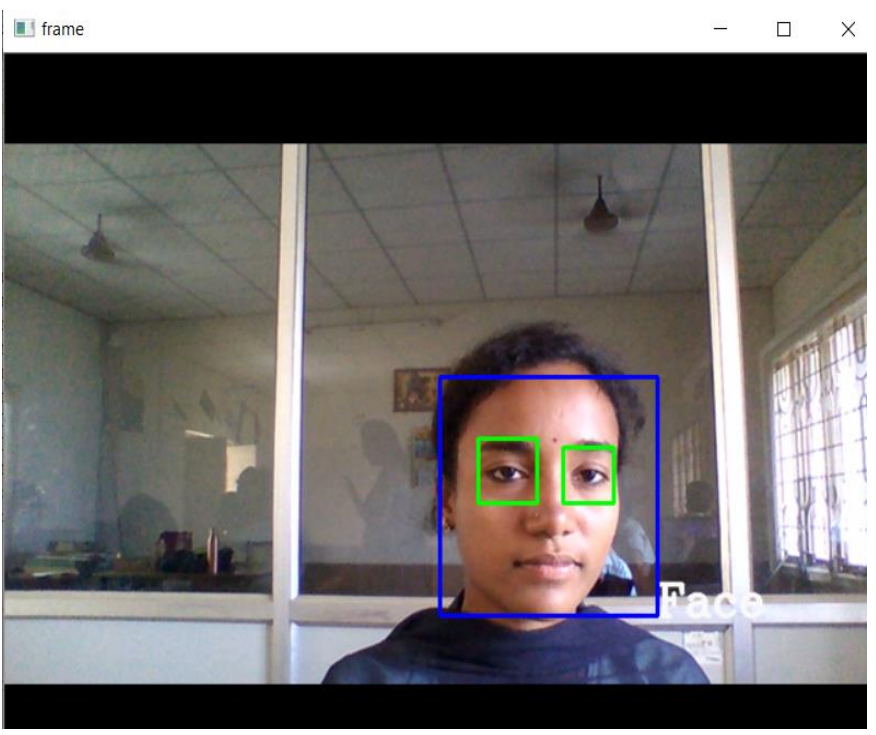

Fig 12. Detect Eye blink and generate alert

\section{CONCLUSION}

This project can be used in every vehicle currently on road to ensure the safety and reduce the chances of an accident due to drowsiness or distraction of driver.This can be implemented using Matlab but, the processing speed in the project using Matlab is very high and the Accuracy implemented by Matlab is less. To overcome these disadvantages the image processing is done by OpenCV which is one of the module in python.The accuracy in image processing using OpenCV is high as well as the processing speed is also high. The advantages using python made this implementation as an easy one. The memory used by the python-opencv is very less.

\section{FUTURESCOPE}

1) This project can be implemented in the form of mobile application to reduce the cost of hardware and to increase the availability of the particular project to the drivers to prevent from accidents.

2) This project can be integrated with car, so that automatic speed control can be imparted if the driver is found sleeping which would be a better way to prevent the accidents.

3) The processing time can be further increased using Artificial Intelligence and accuracy can also be increased using these algorithms.

4) This can be also implemented by sending an message to a particular mobile number when the driver is found to be in drowsiness. This can be helpful for indicating the particular a person of his 


\section{International Journal of Engineering Applied Sciences and Technology, 2020 \\ Vol. 5, Issue 3, ISSN No. 2455-2143, Pages 231-236 \\ Published Online July 2020 in IJEAST (http://www.ijeast.com)}

family member that this person is sleeping. So, that the can also provide an alert or some security to that person.

5) This can also be implemented by adding an GPS module to the project and track the location of that person.

\section{REFERENCES}

[1] Ruian Lui " Design of face detection tracking system " on vol.4 no,,pp.1840,1844,16-18 Image and signal processing (CISP), $20103^{\text {rd }}$ International congress.

[2] Xianghua Fan ,et,al, May 2012. "The system of face detection based on opencv" $20122^{\text {th }}$ chines vol.,pp.648,651,23-25 Control decision conference.

[3] Goel,p, et,al ., "Hybrid approach of Haar cascade classifiers an Geometrical properties of Gender classifer system," Computer sciences(ICCS), 2012 Internationa 1 conference.

[4] Parris, J., et.al, "Face and eye detection on hard datasets," Biometrics (IJCB), 2011 International Joint conference on, vol.,,pp.1,10,11-13 oct-2013.

[5] Peng wang "Automatic Eye Detection and Its validation" on ,vol.,no,pp.164,164,2525 june ,IEEE Computer Society Conference.

[6] Picot, A. et.al., "Online Detection of Drowsiness detection using brain and visual information," on , vol.42, no.3,pp.764,775, part A:Systems and humans, IEEE Transactions.

[7] Xia Liu: Fengliang Xu: Fujimura, k., 2002 IEEE "Real time eye detection and tracking and driver observation under various light conditions," vol.2,no,,pp.3441,351 vol.2, 17-21 June 2002 Intelligent Vehicle Somposium.

[8] Miaou, "Study of Vehicle Scrap page Rates," Oak Ridge National Laboratory, Oak Ridge, TN,,S.P., April 2012.

[9] Wreggit , S.S.,Kim ,C.L., and Weirwille ,W.W., January 2013, Fourth Semi-Annual
Research Report "Research on VehicleBased Driver Status Performance Monitoring", Blacksburg, VA: Virginia Polytechnic Institute and State University, ISE Department.

[10] Bill Fleming, "New Automotive Electronies Technologies"pp.484-488,International Conference on pattern Recognisation.

[11] E. Rogado, J.L. García, R. Barea, L.M. Bergasa, Member IEEE and E. López, February, 2013, "Driver Fatigue Detection System", Proceedings of the IEEE International Conference on Robotics and Biometics, Bangkok, Thailand.

[12] Boon-Giin Lee and Wan-Young Chung, Member IEEE, "Driver Alertness Monitoring Using Fusion of Facial Features and Bio-Signals", IEEE Sensors Journal, VOL. 12, NO. 7, July 2012. 